\title{
Proton resonance elastic scattering in inverse kinematics on the medium heavy nucleus ${ }^{68} \mathrm{Zn}$
}

\author{
N. Imai ${ }^{1, a}$, Y. Hirayama ${ }^{1}$, H. Ishiyama ${ }^{1}$, S.C. Jeong ${ }^{1}$, H. Miyatake ${ }^{1}$, Y.X. Watanabe ${ }^{1}$, H. Makii ${ }^{2}$, S. Mitsuoka ${ }^{2}$, \\ D. Nagae $^{2}$, I. Nishinaka ${ }^{2}$, K. Nishio ${ }^{2}$, and K. Yamaguchi ${ }^{3}$ \\ 1 Institute of Particle and Nuclear Studies, High Energy Accelerator Research Organization (KEK), Oho 1-1, Tsukuba, Ibaraki \\ 305-0801, Japan \\ 2 Japan Atomic Energy Agency, Shirakata Shirane 2-4, Tokai, Ibaraki 319-1195, Japan \\ 3 Institute of Physics, University of Tsukuba, Tennodai 1-1, Tsukuba, Ibaraki 305-8577, Japan
}

Received: 9 July 2010 / Revised: 24 September 2010

Published online: 24 October 2010

(C) The Author(s) 2010. This article is published with open access at Springerlink.com Communicated by S. Kubono

\begin{abstract}
An isobaric analog resonance of ${ }^{69} \mathrm{Zn}$ was studied by the resonance elastic scattering of $p\left({ }^{68} \mathrm{Zn}\right.$, p) with a $5.5 \mathrm{MeV} /$ nucleon ${ }^{68} \mathrm{Zn}$ beam and a thick polyethylene target. The excitation function of the differential cross-section of proton elastic scattering was measured around 0 degrees in the laboratory frame by the thick target inverse kinematics method. The angular momentum, and proton and total widths of the resonance assigned using an $R$-matrix calculation are in good agreement with earlier measurements performed using normal kinematics, demonstrating that the thick target inverse kinematics method is a useful tool for studying the single-particle structures of neutron-rich nuclei.
\end{abstract}

Systematic studies of the neutron single-particle states in neutron-rich nuclei give direct evidence of shell evolution with increasing neutron richness, thereby providing a test of current nuclear-structure models. These states are also important for determining the cross-sections of neutron radiative capture reactions in r-process nucleosynthesis. The recent advent of radioactive ion beam (RIB) facilities provides opportunities for investigating the states of neutron-rich nuclei located far from the stability line by direct reactions.

A straightforward way to study neutron single-particle states in neutron-rich nuclei is the one-nucleon transfer reaction, e.g., $(d, p)$ reaction, in inverse kinematics [1-3]. The final states in these reactions are identified by measuring the proton energy. The spin-parities $\left(J^{\pi}\right)$ and spectroscopic factors are extracted from the angular distributions of differential cross-sections around 0 degrees in the center-of-mass (c.m.) frame. 0 degrees in the c.m. frame corresponds to 180 degrees in the laboratory (lab.) frame, where the energies of protons are often too low to separate the neighboring states, particularly for heavy nuclei which have highly crowded level densities [3]. In order to measure the low-energy protons in wide angular range and to improve the reconstructed energy resolution in the c.m. frame, a large solid-angle silicon semiconductor detectors

\footnotetext{
${ }^{a}$ e-mail: nobuaki.imai@kek.jp
}

(SSDs) array with a high segmentation [4-7] is often employed.

The same spectroscopic information can be obtained by investigating the isobaric analog resonances of the final states through measuring proton resonance elastic scattering at a few points of scattering angles. Under the assumption of isospin symmetry, the neutron single-particle configuration of states in a nucleus ${ }^{A+1} Z$ would be the same as the proton single-particle configuration of states at the high excitation energies in the neighboring nucleus ${ }^{A+1}(Z+1)$, called isobaric analog states. When the ana$\log$ state is above the proton separation energy, the state will be observed in the proton elastic scattering excitation function of a target nucleus ${ }^{A} Z$ as a resonance, called the isobaric analog resonance (IAR). The resonance energy, width, and $J^{\pi}$ can be obtained from the excitation function by $R$-matrix analysis $[8,9]$.

For unstable nuclei, the proton resonance scattering with thick target inverse kinematics (TTIK) is useful [10] for obtaining the excitation function of the proton elastic scattering. At 0 degrees in the lab. frame corresponding to 180 degrees in the c.m. frame, the effect of potential scattering is smallest so that the feature of resonance scattering can be observed to be clearer. Due to the inverse kinematics, the energy of the recoil proton reaches four times higher than the c.m. energy $\left(E_{\text {c.m. }}\right)$. Accordingly, the energy interval between the resonance in the c.m. energy is also mapped to a laboratory energy interval that 
is wider, and largest at 0 degrees where it is four times wider. In addition, the elastic scattering excitation function may be measured over a wide range of energies with the use of a single fixed incident beam energy.

To date, proton resonance elastic scattering using the TTIK method has been extensively used to study proton unbound states within a few $\mathrm{MeV}$ above the proton separation energies $S_{p}$ in light proton-rich nuclei [11-16]. This is in contrast to the study of the neutron singleparticle structure of neutron-rich nuclei, for which the TTIK method has been only applied to the unbound nucleus ${ }^{9} \mathrm{He}$ using the $p\left({ }^{8} \mathrm{He}, p\right)$ reaction. In that work ${ }^{8} \mathrm{He}$ beams of several $\mathrm{MeV}$ in the c.m. [17] were employed.

In this letter, we applied the TTIK method to IARs of the medium-heavy nucleus ${ }^{69} \mathrm{Zn}$ to provide a guide for future experiments using heavy neutron-rich RIBs. We measured the proton resonance elastic scattering using a stable ${ }^{68} \mathrm{Zn}$ beam, allowing us to observe the resonance states in ${ }^{69} \mathrm{Ga}$. The excitation function of proton elastic scattering had been measured in normal kinematics with a step of 10 or $20 \mathrm{keV}$ for incident proton beam energy [18]. Since the resonance at $4.876 \mathrm{MeV}$ in $E_{\text {c.m. }}$, which corresponds to the excited state at $14.9 \mathrm{MeV}$ in ${ }^{69} \mathrm{Ga}$, has a large proton width of $20 \mathrm{keV}$ and there are no other resonances around the state within about $100 \mathrm{keV}$, the resonance is a good candidate as a single resonance for a test of the TTIK method. The resonance can be regarded as an IAR of a bound state of $1 / 2^{+}$at $1.696 \mathrm{MeV}$ in ${ }^{69} \mathrm{Zn}$. Since the respective spins of ${ }^{68} \mathrm{Zn}$ and proton are $0^{+}$and $1 / 2^{+}$, the $J^{\pi}=1 / 2^{+}$resonance state in ${ }^{69} \mathrm{Ga}$ would be strongly favored with the relative angular-momentum transfer of $l=0$. In addition, the inelastic channels ${ }^{68} \mathrm{Zn}(p, p)^{68} \mathrm{Zn}^{*}$ (1.08 MeV, $1.88 \mathrm{MeV}$ ) were observed to be negligibly small compared to the elastic channel in this reaction at 160 degrees in the lab. frame [18] so that we may consider only the elastic channel.

A beam of ${ }^{68} \mathrm{Zn}^{12+}$ with an energy of $5.5 \mathrm{MeV} /$ nucleon, provided by the Japan Atomic Energy Agency (JAEA) tandem accelerator and the superconducting linac, was focused onto a polyethylene target with a thickness of $3.2 \pm 0.2 \mathrm{mg} / \mathrm{cm}^{2}$. The beam spot size was $2 \mathrm{~mm}$ in diameter. The output energy at the exit of the target was calculated to be $2.9 \mathrm{MeV} /$ nucleon using the formula with the low-energy correction of ref. [19]. The target was backed by an $10.8 \pm 0.5 \mathrm{mg} / \mathrm{cm}^{2}$ thick carbon sheet to stop the beam. The energies of the recoil protons were measured using three layers of $300 \mu \mathrm{m}$ thick pad-type SSDs of $48 \times 48 \mathrm{~mm}^{2}$ (HAMAMATSU S4276-02) located $50 \mathrm{~cm}$ downstream of the target at 0 degrees in the lab. frame. The energies of the protons were degraded by a $1.48 \pm 0.02 \mathrm{~mm}$ thick aluminum plate located just downstream of the carbon sheet. The degrader allowed us to measure the resonance around $E_{\text {c.m. }}=5 \mathrm{MeV}$ by the SSDs. The size of degrader was $1.5 \times 3 \mathrm{~cm}^{2}$ which was large enough to intercept trajectories of the reaction products to the SSDs from the target. In addition, we placed a $2.5 \mathrm{~mm}$ thick plastic scintillator of $50 \times 50 \mathrm{~mm}^{2}$ just behind the SSDs as a veto counter. In the experiment, protons relevant to the carbon in the polyethylene were evaluated by carrying out the measurement with only the carbon sheet. The beam intensities

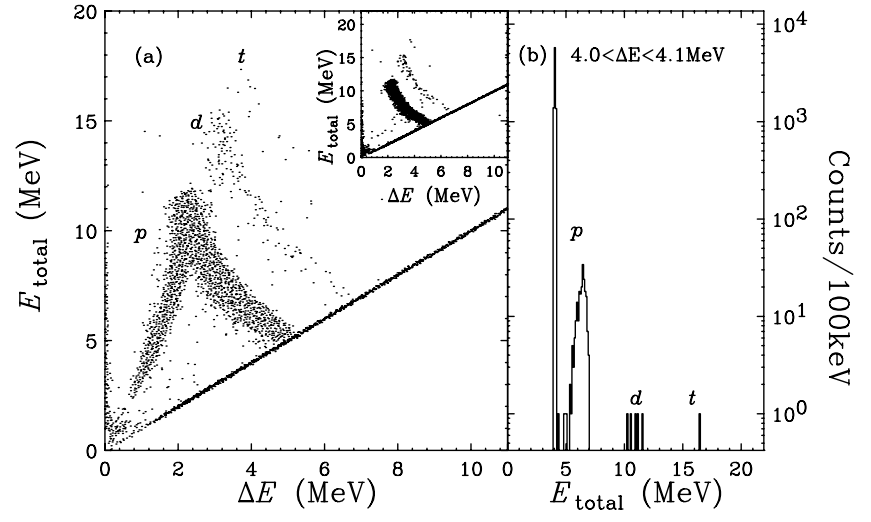

Fig. 1. (a) $\Delta E-E_{\text {total }}$ correlation measured by SSDs. Protons, deuterons, and tritons are indicated by " $p$ ", " $d$ ", and " $t$ " beside the respective loci. The correlation of particles which did not hit the veto counter is presented in the inset. (b) Spectrum of $E_{\text {total }}$ for $4.0<\Delta E<4.1 \mathrm{MeV}$. See the text for details.

were measured by a Faraday cup $10 \mathrm{~m}$ upstream of the target at the beginning of the every beam run. The typical intensity was about $8 \mathrm{epA}$. The fluctuation of the intensity in each two-hours run was observed to be within $10 \%$.

Figure 1(a) presents the correlation between the energy loss in the first SSD $(\Delta E)$ and the sum of the energy losses in all the SSDs $\left(E_{\text {total }}\right)$. Protons could be clearly distinguished from other particles, i.e., deuterons and tritons. Events which punched through the SSDs were excluded from the analysis by using the veto signals as presented in the inset of fig. 1(a). Note that many of the charged particles which stopped in the first SSD were observed, as shown in fig. 1(b). Because they were detected both with the polyethylene and the carbon targets, they should be reaction products of the beams with the carbon. When we selected the events of $4.0<\Delta E<4.1 \mathrm{MeV}$, the yield at $E_{\text {total }}=4 \mathrm{MeV}$ is larger than the proton yield at $6 \mathrm{MeV}$ by two orders of magnitudes. Their atomic numbers appear to be larger than $Z=1$ because of the larger energy losses. To distinguish the protons stopped in the first SSD, further experimental information is required, such as the time of flight (TOF) of the particles. Unfortunately, no additional suitable parameters were recorded, and hence our analysis is limited to the excitation functions of protons that passed through the first SSD.

Monte Carlo simulations were performed to estimate the experimental energy resolution, the detection efficiency of the SSDs, and the conversion function of the measured $E_{\text {total }}$ to $E_{\text {c.m. }}$. The simulations included the effect of energy loss and multiple scattering in the materials, and the energy resolutions of the detectors. In the simulations, we tuned several parameters to reproduce the following two aspects of the shape of the $\Delta E-E_{\text {total }}$ correlation. The first was the bending points at $\left(\Delta E, E_{\text {total }}\right)=$ $(5 \mathrm{MeV}, 5 \mathrm{MeV})$ and $(2.2 \mathrm{MeV}, 12 \mathrm{MeV})$, where protons punched through the first and the third layers, respectively. The thicknesses of the dead layers of SSDs were adjusted to reproduce these points. The second aspect was the experimental energy resolution which was reproduced by introducing an energy-dependent resolution, mainly 


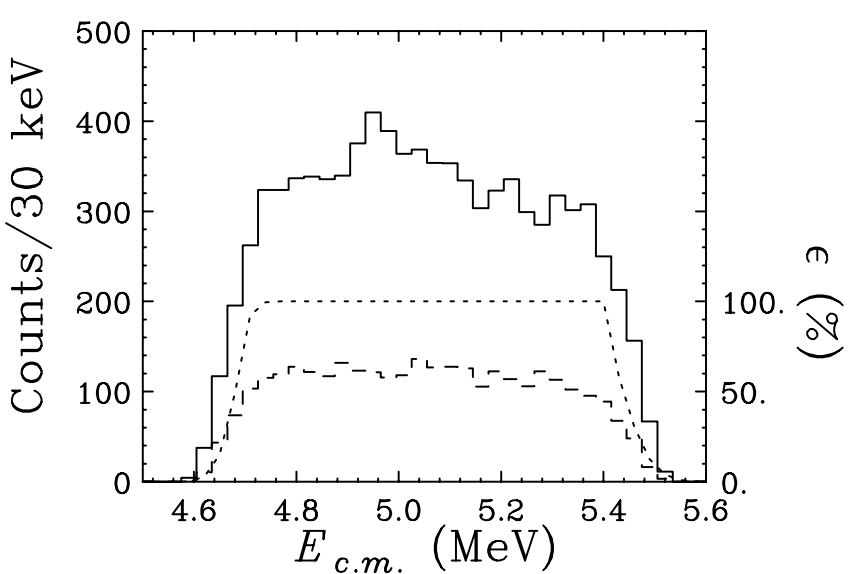

Fig. 2. Yields of protons measured with the polyethylene (solid line) and carbon (dashed line) targets as a function of $E_{\text {c.m. }}$. for protons stopped in either the second or third SSD. The dotted line indicates the simulated detection efficiency $(\epsilon)$.

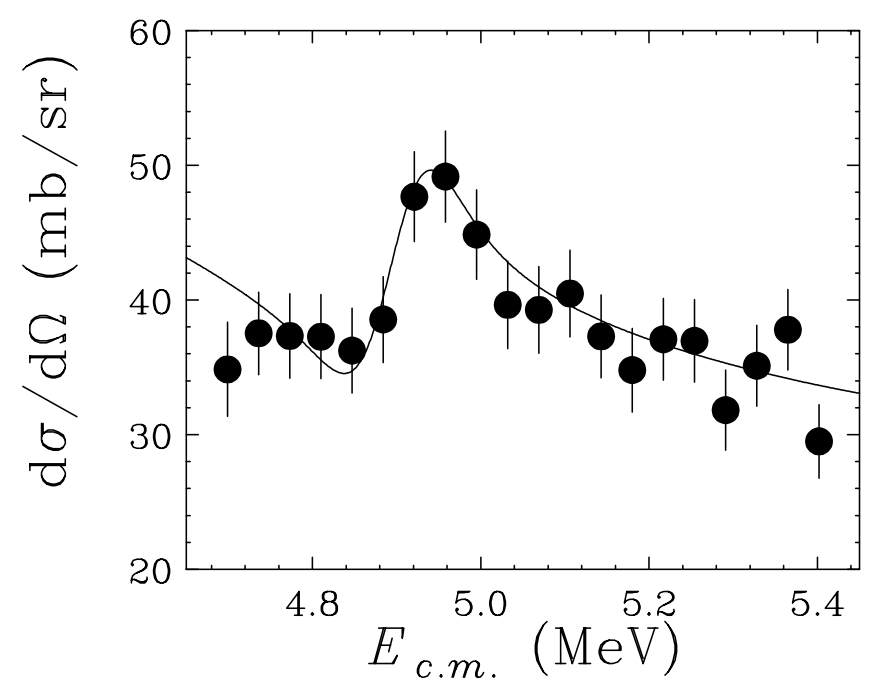

Fig. 3. Excitation function of the differential cross-section at 0 degrees in the lab. frame. The solid line indicates the fitted $R$-matrix calculation assuming $l=0$.

due to the multiple scattering in the aluminum plate, about $200 \mathrm{keV}(1 \sigma)$ for $E_{\text {total }}=9 \mathrm{MeV}$, corresponding to an energy resolution of $30 \mathrm{keV}$ for $E_{\text {c.m. }}=5 \mathrm{MeV}$.

The energy distributions of protons observed with the carbon and the polyethylene target are compared in fig. 2 . The yield of protons measured with the carbon target was normalized using the relative beam dose. The protons associated with the carbon in polyethylene were found not to be negligible. These protons can be emitted from the compound system by evaporation, as suggested by the flat distribution in the c.m. frame.

The excitation function of the differential cross-section of the proton elastic scattering at $\theta_{\text {lab }} \simeq 0$ degrees is shown in fig. 3 . The cross-section was obtained by taking into account the detection efficiency of the SSDs as illustrated by the dotted line in fig. 2. Because the readout of the Faraday cup was not designed for a beam current of as low as several epA, the absolute value is not reliable, al- though the relative beam intensity which was used for the normalization of the measurements with the carbon target may be reliable. Hence, we normalized the experimental data to the theoretical cross-sections at off-resonance region, $E_{\text {c.m. }}<4.8 \mathrm{MeV}$, as described in ref. [18]. In the excitation function, the resonance of ${ }^{69} \mathrm{Ga}$ was clearly observed.

The proton and total widths, $\Gamma_{p}$ and $\Gamma_{\text {total }}$, and resonance energy $E_{\text {reso }}$ were investigated assuming the angular momentum $l$ of the resonance by fitting the $R$ matrix calculation to the obtained excitation function with MINUIT [20]. In the calculation, the potential scattering was obtained using the global optical model potential set [21] as used in ref. [18]. The $R$-matrix calculation including the experimental energy resolution was fitted to the experimental data from $E_{\text {c.m. }}=4.7$ to $5.2 \mathrm{MeV}$.

The minimum value of $\chi^{2}$ was 5.5 with 12 degrees of freedom. The resultant parameters were $\Gamma_{p}=16.3 \pm$ $8.1 \mathrm{keV}, \Gamma_{\text {total }}=58.2 \pm 45.6 \mathrm{keV}$, and $E_{\text {reso }}=4899 \pm$ $12 \mathrm{keV}$. Here the uncertainties were derived from only the statistical error. The systematic error was estimated to be $6 \%$ by taking a quadratic sum of the uncertainties derived from the ambiguities in the relative normalization $(4 \%)$ and in the proton energies $(4 \%)$. The values obtained are in good agreement with the parameters $\left(\Gamma_{p}, \Gamma_{\text {total }}, E_{\text {reso }}\right)=(20 \mathrm{keV}, 60 \mathrm{keV}, 4873 \mathrm{keV})$ previously measured in normal kinematics [18]. When we sought the parameters assuming $l=1,2$, they did not converge, ruling out these two angular momenta. The best fitted curve assuming $l=0$ is presented in fig. 3 .

The spectroscopic factor $S$ was deduced from $\Gamma_{p}$ using the formula [9]

$$
S=\left.\frac{(N-Z+1) 2 \mu r \Gamma_{p}}{2 P_{c}^{0} e^{-2 \delta^{I_{l j}}} \hbar^{2} u_{n}^{2}(r)}\right|_{r=a_{c}},
$$

where $N$ is the number of neutrons in the target nucleus, $\mu$ is the reduced mass of the proton, $P_{c}^{0}$ is the optical penetrability, $a_{c}$ is the matching radius, and $u_{n}(r)$ is the single-particle wave function of a bound-state neutron in the parent state of $(l, j)$. The neutron wave function $u_{n}(r)$ was obtained by solving the Schrödinger equation to reproduce the neutron binding energy of the parent state. $S$ was deduced to be $0.39 \pm 0.19$. The large spectroscopic factor suggests that the resonance state should be the isobaric analog resonance of ${ }^{69} \mathrm{Zn}$. The obtained $S$ is consistent with the value of $S=0.33$ determined from the $(d, p)$ reaction [22].

In summary, we have studied the proton resonance elastic scatting in inverse kinematics using a stable beam of ${ }^{68} \mathrm{Zn}$ with a thick polyethylene target. The detection and identification of protons from the reaction enabled the study of the resonance states in ${ }^{69} \mathrm{Ga}$. The excitation function of the protons shows distinctive structures depending on the orbital angular momentum specific to the resonance. Assuming the isospin symmetry, the resonance can be considered as the isobaric analog resonance of the neutron single-particle state of ${ }^{69} \mathrm{Zn}$. An $R$-matrix analysis was performed and the spectroscopic factor, angular momentum, and resonance energy were extracted. The results 
are in good agreement with the earlier measurement of isobaric analog resonances. The spectroscopic factor is also consistent with the value obtained from the $(d, p)$ reaction in normal kinematics. Considering the simple experimental setup, the present work demonstrates that the proton resonance elastic scattering with the TTIK method for IARs can be a powerful tool for studying neutron singleparticle states in neutron-rich nuclei.

We would like to thank the staff of the JAEA tandem accelerator. This work was funded in part by a Grant in-Aid for Science Research (A) from the Japan Ministry of Education, Culture, Sports, and Technology under the contract number 20244036.

Open Access This article is distributed under the terms of the Creative Commons Attribution Noncommercial License which permits any noncommercial use, distribution, and reproduction in any medium, provided the original author(s) and source are credited.

\section{References}

1. G. Kraus et al., Z. Phys. A 340, 339 (1991).

2. K.L. Jones et al., Phys. Rev. C 70, 067602 (2004).
3. J.S. Thomas et al., Phys. Rev. C 71, 021302 (2005).

4. Y. Blumenfeld et al., Nucl. Instum. Methods A 421, 471 (1999).

5. T. Davinson et al., Nucl. Instum. Methods A 454, 350 (2000).

6. M.S. Wallace et al., Nucl. Instum. Methods A 583, 302 (2007).

7. M. Labiche et al., Nucl. Instum. Methods A 614, 439 (2010).

8. A.M. Lane, R.G. Thomas, Rev. Mod. Phys. 30, 257 (1958).

9. W.J. Thompson, J.L. Adams, D. Robson, Phys. Rev. 173, 975 (1968).

10. K.P. Artemov et al., Sov. J. Nucl. Phys. 52, 1460 (1990).

11. R. Coszach et al., Phys. Rev. C 50, 1695 (1994).

12. L. Axelsson et al., Phys. Rev. C 54, R1511 (1996).

13. D.W. Bardayan et al., Phys. Rev. Lett. 83, 45 (1999).

14. G.V. Rogachev et al., Phys. Rev. C 64, 061601 (2001).

15. T. Teranishi et al., Phys. Lett. B 556, 27 (2003).

16. C. Ruiz et al., Phys. Rev. C 71, 025802 (2005).

17. G.V. Rogachev et al., Phys. Rev. C 67, 041603(R) (2003).

18. W.S. Steiner et al., Phys. Rev. C 4, 1684 (1971).

19. S.P. Ahlen, Phys. Rev. A 17, 1236 (1978).

20. CERN program library.

21. F.D. Becchetti jr., G.W. Greenless, Phys. Rev. 182, 1190 (1969).

22. J.A. Bieszk, J.A. Thomson, Nucl. Phys. A 359, 269 (1981). 